\title{
NUEVOS TERRITORIOS EN ENTORNOS DE PROGRAMAS HABITACIONALES PÚBLICOS. ASENTAMIENTO INFORMAL PARQUE ITUZAINGÓ ANEXO II EN CÓRDOBA, ARGENTINA
}

\author{
NEW TERRITORIES IN PUBLIC HOUSING PROGRAM ENVIRONMENTS. INFORMAL SETTLEMENT \\ PARQUE ITUZAINGÓ ANEXO II IN CÓRDOBA, ARGENTINA
}

\author{
Ana Laura Elorza ${ }^{1}$ \\ Maria Cecilia Marengo² \\ Maria Virginia Monayar ${ }^{3}$ \\ Maria Florencia Sosa ${ }^{4}$
}

\section{RESUMEN}

Las cuestiones urbanas en el siglo XXI, señalan como aspectos centrales la lógica mercantilista en el desarrollo urbano y la intensificación de las desigualdades sociales. La política habitacional pública no escapa a esta lógica, observándose localizaciones cada vez más distantes e incremento de los asentamientos informales. El trabajo expone la forma en que ésta, sin proponérselo, ha generado condiciones para la producción de nuevos territorios periféricos informales que se han configurado a partir de su materialización y reflexiona sobre el derecho a la ciudad en este contexto. Metodológicamente, para analizar los procesos y transformaciones espaciales derivados de la

\footnotetext{
${ }^{1}$ Dra. en Ciencias sociales (UNCuyo), Mgter. en Gestión y Desarrollo Habitacional, Lic. en Trabajo Social. Investigadora Adjunta CONICET en Instituto de Investigación de Vivienda y Habitat de la Facultad de Arquitectura, Urbanismo y Diseño y docente y extensionista de la Facultad de Ciencias Sociales de la Universidad Nacional de Córdoba. CONICET- Instituto de Investigación de Vivienda y Habitat de la Facultad de Arquitectura, Urbanismo y Diseño de la Universidad Nacional de Córdoba - Argentina. ORCID Id: https://orcid.org/0000-00017099-6307 E-mail: analauraelorza@unc.edu.ar

2 Ph.D, Universidad Tecnológica de Delft - Holanda.Arquitecta, Magister en Diseño Arquitectónico y urbano, FAUD - Universidad Nacional de Córdoba Argentina. Investigadora Independiente, CONICET en el Instituto de Investigación de Vivienda y Hábitat FAUD, Gv. a IDH-UNC/ CONICET. Directora del Instituto de Investigación de Vivienda y Hábitat (INVIHAB-FAUD UNC) de la Facultad de Arquitectura, Urbanismo y Diseño, Universidad Nacional de Córdoba. Profesora Titular FAUD - UNC. Directora de la Carrera de Doctorado en Arquitectura (DoctA) FAUD - UN. CONICET- Instituto de Investigación de Vivienda y Habitat de la Facultad de Arquitectura, Urbanismo y Diseño de la Universidad Nacional de Córdoba - Argentina. CORCID Id: https://orcid.org/0000-00015670-5390 E-mail: mcmarengo@unc.edu.ar

${ }^{3}$ Dra. en Ciencias Sociales con mención en geografía. Investigadora Asistente CONICET- Instituto de Investigación de la Vivienda y Hábitat (FAUD-UNC), docente de la Facultad de Ciencias Sociales de la Universidad Nacional de Córdoba. CONICET- Instituto de Investigación de Vivienda y Hábitat de la Facultad de Arquitectura, Urbanismo y Diseño de la Universidad Nacional de Córdoba. - Argentina. Email: virginia.monayar@unc.edu.ar

${ }^{4}$ Becaria doctoral CONICET - Instituto de Investigación de la Vivienda y Hábitat (FAUD-UNC), doctoranda en el Doctorada de Arquitectura FAUD-UNC. CONICET- Facultad de Arquitectura, Urbanismo y Diseño de la Universidad Nacional de Córdoba. - Argentina. E-mail: arq.florenciasosa@gmail.com
} 
localización, se desarrolló un abordaje cuali-cuantitativo del programa habitacional masivo Mi Casa Mi Vida, en la ciudad de Córdoba, Argentina. Posteriormente, se trabajó un estudio de caso, con el relevamiento de una ocupación informal buscando comprender, desde un enfoque micro, las condiciones habitacionales y de vida de los habitantes. Los resultados dan cuenta de la forma de producción de los territorios informales periféricos y aportan reflexiones sobre la evolución de los entornos próximos a las urbanizaciones de producción estatal. Muestran las relaciones de proximidad física y social en situaciones de informalidad, en las formas de acceso al suelo y la vivienda, vinculando la producción del asentamiento y la política habitacional pública.

Palabras clave: política habitacional, informalidad, condiciones habitacionales, periferia, derecho a la ciudad

\section{ABSTRACT}

The urban question in the XXI century, indicates as the central issue the mercantilist logic in urban development and the intensification of social inequalities. Public housing policy does not escape this logic, observing increasingly distant locations and an increase in informal settlements. The paper accounts for the way in which it, without intending to, has generated conditions for the production of new informal peripheral territories, that have been configured since its materialization and reflect on the right to the city in this context. Methodologically to analyze the spatial processes and transformations derived from location, a qualitative-quantitative approach was developed to the massive housing program Mi Casa Mi Vida, in Córdoba city, Argentina. Subsequently, a case study was carried out, with the census of an informal occupation seeking to understand, from a micro perspective, the living and housing conditions of the inhabitants. The results show the way informal peripheral territories are produced and provide reflections on the evolution of the surroundings public housing urbanizations. They show the relationships of physical and social proximity in situations of informality, in the forms of access to land and housing, linking the production of the settlement and the public housing policy.

Keywords: housing policy, informality, housing conditions, periphery, right to the cit 


\section{INTRODUCCIÓN Y PRESENTACIÓN DEL ESTUDIO}

Un aspecto central en las nuevas cuestiones urbanas del siglo XXI son las desigualdades sociales, que se intensifican en un proceso de mercantilización del desarrollo urbano y muchos autores señalan como injusticia espacial (SECCHI, 2015; SOJA, 2014). En las últimas tres décadas, las ciudades han sufrido transformaciones vinculadas a la reestructuración del sistema capitalista, basadas en el sistema financiero y su transnacionalización, caracterizando un modelo de desarrollo urbano neoliberal, con fuertes implicancias en sus territorios (JANOSCHKA y HIDALGO, 2014). ROLNIK (2018) sostiene que la financiarización de la economía, ocupa las ciudades y se materializa físicamente en "paisajes para la renta", reconoce las particularidades que va asumiendo en cada localidad, según las economías y las políticas específicas en cada tiempo y espacio. En las ciudades latinoamericanas, estos paisajes son implantados en áreas identificadas como espacios de residencia y consumo de las élites, que en muchas oportunidades rompen espacios reconocidos o se constituyen como fronteras, en relación a los "paisajes para la vida", construidos desde la lógica de la supervivencia, de las necesidades y los deseos de prosperidad, producidos con escasos recursos y en terrenos vacantes (como las periferias de bajo valor y las áreas en riesgo ambiental, entre otros).

La lógica mercantilista condiciona las posibilidades de acceso a suelos urbanizados, el espacio se vuelve un bien escaso que involucra prácticas especulativas y se produce un deterioro de su calidad en un sentido amplio, vinculado con el deterioro de la vida urbana y con el derecho a la ciudad (LEFEBVRE, 1970 citado en DE MATTOS, 2015). Como resultado, se ha generado un espacio periférico altamente fragmentado, donde conviven barrios internamente homogéneos, pero desiguales entre sí, en un territorio urbanizado desarticulado. La política de vivienda no escapa a esta lógica, a la exacerbación de los procesos especulativos de valorización del suelo urbano a través de la expansión de mercados formales e informales, que algunos autores señalan como extractivista (SVAMPA y VIALE, 2014). Prueba de ello es la localización cada vez más distante de los conjuntos habitacionales públicos, en sectores periféricos con dificultades en la provisión y acceso a servicios de calidad y a las áreas de centralidad, (que se configuran como posibilidades para el aprovechamiento de los recursos urbanos, trabajo, educación, salud, etc.); lo cual representa una tensión en relación al derecho a la ciudad, en tanto acceso a los recursos urbanos y al ejercicio de participar en el proceso de urbanización (HARVEY, 2013).

En la ciudad de Córdoba (Argentina) durante el período 2003-2010 se desarrolló una ambiciosa política habitacional que tuvo como objeto erradicar las villas en situación de riesgo ambiental. Muchas se localizaban en áreas centrales o pericentrales que habían sufrido inundaciones, o en sectores que 
fueron afectados a la realización de obras públicas (donde posteriormente se localizaron proyectos residenciales o comerciales promovidos por el mercado inmobiliario y dirigidos a sectores de ingresos medios o medio-altos). Los proyectos de 12.000 viviendas sociales unifamiliares que construyeron conformaron conjuntos de distintas escalas y se materializaron mayoritariamente en áreas periféricas, con escasa integración urbana y servicios de baja calidad.

A más de 10 años de su ejecución, estos territorios han transformado su morfología y se reconocen cambios a nivel barrial derivados del propio proceso de consolidación, así como en las unidades de vivienda que los integran. Las estrategias habitacionales de las familias adjudicatarias, se orientaron a la ampliación del núcleo inicial (dado las mínimas superficies de metros cuadrados cubiertos provistas en el programa) y a la construcción de nuevos espacios habitables en los lotes. El dato significativo, es que se modificaron los entornos urbanos de estos barrios como resultado de las estrategias colectivas de toma de tierra y de los procesos de autoproducción del hábitat por parte de habitantes vinculados a los conjuntos habitacionales públicos.

En este trabajo analizamos la forma en que la política habitacional sin proponérselo, ha generado condiciones para la producción de nuevos territorios periféricos informales, aprovechando las condiciones de cercanía, la incipiente urbanización, la extensión de redes de servicios e infraestructura y la existencia de suelo vacante próximo, entre otros aspectos. Hacemos foco en la construcción de los espacios informales reconociendo, desde un enfoque micro, las condiciones habitacionales y de vida de quienes los habitan. Por otra parte, buscamos dar cuenta de las relaciones de proximidad físicas y sociales imbricadas en la producción de un asentamiento informal y reflexionar sobre la evolución de los entornos residenciales próximos a las urbanizaciones estatales donde se reproduce esta lógica, reflexionando sobre el derecho a la ciudad

Metodológicamente, realizamos un abordaje cuanti- cualitativo, a partir de la triangulación de métodos. En una primera etapa, analizamos los procesos y transformaciones espaciales derivados de la localización de la política habitacional pública en la ciudad de Córdoba, identificando los momentos y sucesos de producción residencial en los entornos de los conjuntos habitacionales. Se utilizaron fuentes de información satelital de Google Earth, Google Maps y sus herramientas de aplicación. Posteriormente desarrollamos un estudio de caso, de una ocupación informal localizada en terrenos colindantes a la urbanización estatal "Ciudad de Mis Sueños", denominada "Parque Ituzaingó II". Se realizó un relevamiento sobre el total de las familias residentes, aplicando una encuesta relativa a las condiciones sociales y habitacionales.

Los resultados dan cuenta de las estrategias que se ponen en juego para resolver la cuestión de hábitat (acceso al suelo y la vivienda), de las condiciones habitacionales resultantes, así como de la 
carencia de respuestas por parte del Estado frente a las dificultades y complejidad del problema. También, se discute la forma de producción de estos territorios y los efectos derivados de la implementación de políticas habitacionales focalizadas que reproducen condiciones de fragmentación en los espacios periféricos.

La comprensión de qué tipo de entornos físicos y sociales se conformaron donde se localizan los conjuntos de viviendas materializados por la intervención estatal, nos permite caracterizar las transformaciones que tienen lugar y aportan insumos para la planificación del crecimiento urbano. Finalmente, contribuyen a reflexionar sobre la efectividad de las políticas habitacionales, siendo indispensable reconocer los principios del derecho a la ciudad como horizonte de equidad en el desarrollo urbano de la ciudad.

\section{LA MERCANTILIZACIÓN DEL SUELO Y LA PRODUCCIÓN DE VIVIENDA ESTATAL}

La vivienda social sigue siendo un elemento constitutivo de las ciudades latinoamericanas. En el último período, de cara al proceso de mercantilización del desarrollo urbano, las políticas públicas habitacionales implementadas por los diferentes estados de la región, no escapan a este contexto. Las instancias gubernamentales se han convertido en organismos financieros que funcionan preponderantemente como administradores de créditos, para la construcción de la vivienda social. El efecto común de estas políticas ha sido la expansión masiva de grandes conjuntos con menor o mayor grado de integración urbana, principalmente localizados en las periferias de las ciudades, condicionados por la renta del suelo y el comportamiento especulativo de los mercados (ZICCARDI, 2015; CARRIÓN y HANLEY, 2005; HIDALGO DATTWYLER, 2019).

Numerosas investigaciones de la región han tenido como objeto el análisis de los impactos de las políticas habitacionales en las condiciones de vida y en las modalidades de producción de la ciudad y los espacios residenciales. Por un lado, se han evaluado los resultados de las políticas como respuesta a la necesidad habitacional desde la perspectiva y subjetividades de la población adjudicataria y se reconocen, en las últimas décadas, mejoras en las condiciones de vivienda respecto de las que tuviera anteriormente (ZICCARDI, 2015; MARENGO y ELORZA, 2018). Por otro lado, en diversos estudios, se abordan cuestiones como la respuesta predominantemente cuantitativa en el abordaje del déficit habitacional, la desvinculación de la producción habitacional con las orientaciones y políticas para el desarrollo urbano, el rol de los organismos de financiamiento internacional en la promoción masiva de viviendas y la mercantilización de las mismas expresadas en la urbanización de suelo periférico y distante, con altas 
tasas de ganancias para los desarrolladores inmobiliarios (RODRÍGUEZ y SUGRANYES, 2005; ZICCARDI, 2015; MARENGO y ELORZA, 2018; HIDALGO DATTWYLER, 2019; CARRIÓN, 2019).

Este sistema de vivienda masiva y mercantilizada, ha dado lugar en muchos casos a lo que algunos autores llaman "gente sin casa y casas sin gente" (ZICCARDI, 2016), "la vivienda privada de ciudad" (RODRÍGUEZ y RODRÍGUEZ, 2016), o “precariópolis” (HIDALGO DATTWYLER, 2007, 2019). En estos análisis prevalecen la crítica a la producción de urbanizaciones periféricas con grandes extensiones, y los impactos en términos de los entornos construidos, la falta de oportunidades de empleo y de acceso a equipamientos, como también los costos en la extensión de servicios urbanos que deben asumir los gobiernos locales, entre otros aspectos. Expresión de esos costos son, por ejemplo, la cantidad de vivienda vacía en los conjuntos habitacionales en México (ZICCARDI, 2015) o las precarias soluciones habitacionales de "los con techo" en Chile (RODRÍGUEZ y SUGRANYES, 2005).

En América Latina las políticas habitacionales que se han formulado para atender la demanda y el déficit habitacional en los últimos años, no han dado cuenta de un abordaje integral de la cuestión; han priorizado la construcción de viviendas nuevas en localizaciones periféricas y de baja calidad, antes que revisar las causas estructurales que están detrás del acceso desigual al mercado del suelo urbano. Respecto al déficit habitacional, en Argentina, si bien los datos censales (2001-2010) permiten observar una mejora relativa, en términos absolutos se mantienen las carencias de una buena parte de la población que comparte vivienda con otros hogares o reside en viviendas deficitarias en cuanto a la habitabilidad, mientras que una importante cantidad de viviendas permanece desocupada (REESE y CATENAZZI, 2016).

En este marco, cobra importancia un abordaje integral entre las políticas de vivienda y suelo, entre la calidad habitacional y de los entornos. En la Nueva Agenda Urbana aprobada en la Conferencia Hábitat III en Quito en el año 2016, se hace énfasis en cómo el lugar de residencia para la población de bajos ingresos, representa mecanismos de reproducción de situaciones de pobreza y de desigualdades territoriales y señala el rol de la política pública en cuanto a la localización urbana, escala de intervención y conformación social de la población destinataria de los conjuntos habitacionales (Marengo y Elorza, 2018). En esa línea, ACIOLY (2018) plantea la necesidad que las políticas habitacionales consideren la "vivienda al centro" de localizaciones que promuevan una mejor estructura de oportunidades, como proceso que posibilitaría la efectivización del derecho a la ciudad.

El acceso a la vivienda se produce fundamentalmente a través de la dinámica y lógica del mercado inmobiliario formal o por el subsidio estatal a través de las políticas habitacionales públicas (según el nivel de ingresos del grupo social), y quienes no logran resolver la cuestión habitacional por ninguno de estos sistemas, producen otras modalidades de acceso. En este sentido, se reconoce la necesidad de profundizar el análisis sobre este tipo de producción de territorialidades; por un lado, como procesos de 
autoproducción del hábitat (ORTIZ, 2007) y por otro, como impacto devenido de las formas y lógicas de la propia política habitacional, como nos proponemos desarrollar en este artículo.

\section{LA AUTOPRODUCCIÓN DEL HÁBITAT INFORMAL: "PAISAJES PARA LA VIDA"}

A los fines analíticos en este trabajo, se focaliza en los procesos de autoproducción del hábitat informal desde la lógica de la necesidad (ABRAMO, 2012), que refiere a la producción que se realiza bajo iniciativa y control de los propios pobladores, de manera: individual/familiar o comunitaria, colectiva y organizada. Frente a la imposibilidad de acceder al hábitat mediante el mercado formal o a través de políticas públicas, los habitantes organizados de manera colectiva comienzan el proceso de construir un lugar para la reproducción cotidiana. "Paisajes para la vida", en términos del ROLNIK (2018), producidos con escasos recursos, en terrenos ociosos y, usualmente de bajo valor económico, dando lugar a urbanizaciones populares de origen informal. En general, los territorios resultantes se caracterizan por la precariedad en sus condiciones habitacionales y pueden considerarse como un "espacio negociado" (DUHAU y GIGLIA, 2008). Son la resultante de disputas y acuerdos, por un lado, con otros actores (el Estado, las empresas de servicios públicos, las empresas inmobiliarias, etc.), con los que se negocian aspectos vinculados a la permanencia o al desalojo de los terrenos, el acceso a servicios públicos, el reconocimiento de las demandas, etc.; y por otro lado, al interior del grupo de las familias y la organización socio territorial (en la definición de los lotes, la organización para la construcción de redes de infraestructura, estrategias de resistencia, pautas de convivencia, etc.) (ELORZA y MORILLO, 2017).

Estos territorios, se reproducen en el espacio urbano a pesar de las acciones asumidas por los gobiernos en materia de política habitacional, incluso aquellas destinadas a mitigar, regular o regularizar los procesos de autoproducción habitacional. Asimismo, su sostenida reproducción da cuenta tanto del déficit y la demanda de suelo y vivienda, como de la masiva capacidad de autoproducción de los sectores populares, para construir y consolidar las áreas de la ciudad que habitan (RODRÍGUEZ, et. al., 2007). Uno de los grupos más afectados son las nuevas generaciones de familias jóvenes (MARENGO y ELORZA, 2018; MONAYAR, 2018) que reproducen las condiciones de inaccesibilidad que tuvieron sus padres, aun cuando éstos lograron ser adjudicatarios de una vivienda implementada a través de políticas habitacionales.

La persistencia en las dificultades de acceso a suelo urbano formal, el crecimiento de las familias y allegados en las viviendas, las modificaciones demográficas en cuanto a la composición de los hogares, las preferencias de localización en relación a la proximidad a redes de sociales y de supervivencia (familiares y vecinales) y la falta de respuestas estatales eficientes y dinámicas para abordar la demanda 
habitacional (que obtiene en la informalidad la única alternativa de acceso a la vivienda), son algunos motivos que mantienen la producción y reproducción de estos territorios en las ciudades de América Latina.

Si bien cada región, país y localidad presentan singularidades (villas, favelas, barriadas, campamentos, tomas, ocupaciones, etc.), se identifican características comunes como las descriptas. Particularmente en Argentina, históricamente fueron las villas de emergencia la tipología de ocupación informal predominante, aunque a partir de los '80 esto empieza a cambiar dando lugar a otras modalidades de ocupación del espacio como los loteos fraudulentos y las tomas de tierra (CRAVINO, 2006). A diferencia de las villas, la construcción de los territorios en las tomas se realiza de manera colectiva y organizada. La decisión de una localización definitiva, le confiere cierta organización semejante y con vinculaciones a la estructura urbana del entorno y una rápida dinámica de consolidación en la que a veces el Estado muestra cierta "política de tolerancia" dadas la falta de respuesta a las demandas (MONAYAR et al, 2019). Como plantea ROY (2013), en muchos casos el propio Estado opera de manera informalizada, ganando una flexibilidad territorializada en la que no se cuenta plenamente con los mecanismos meramente formales de acumulación y legitimación, lo cual "significa que la informalidad no es un dominio desregulado, sino estructurado a través de varias formas de regulación extralegal, social y discursiva" (p. 168).

Estas condiciones refuerzan otros postulados que indican que frente a esta problemática el Estado actúa mediante la relocalización; la reurbanización; el desalojo o el "dejar hacer". Cada una de estas alternativas representan las formas en que se interpreta el derecho a la ciudad y el problema del acceso al suelo y vivienda, en tiempos y espacios determinados, las propuestas programáticas para resolverlo y los sujetos definidos como destinatarios.

\section{EL PROGRAMA MI CASA MI VIDA EN LA CIUDAD DE CÓRDOBA Y LA REPRODUCCIÓN URBANA INFORMAL}

En la ciudad de Córdoba (Argentina), durante el periodo 2003-2010, el gobierno provincial ejecutó una política pública habitacional, el programa "Mi Casa, Mi Vida", cuyo objetivo era erradicar las villas en riesgo ambiental por estar asentadas en áreas inundables. Como resultado, se erradicaron 70 villas a 39 nuevos barrios, en su totalidad con localizaciones en áreas periféricas y en la mayoría de los casos, aisladas de la mancha urbana consolidada. Estas áreas, inicialmente destinadas al uso rural/industrial, requirieron 
un cambio de patrón de uso del suelo a urbano/residencial. La modificación afectó sólo a las tierras donde se localiza la urbanización y no a los terrenos colindantes (MONAYAR, 2018), lo que ha generado, en la mayoría de los casos, una situación conflictiva por la colindancia de usos incompatibles, y grandes sectores vacantes que dificultan la provisión de servicios.

Los conjuntos habitacionales responden a una misma tipología organizativa, manzanas con lotes uniformes de entre $250 \mathrm{~m} 2$ y $300 \mathrm{~m} 2$ y una única tipología de vivienda seriada y estandarizada de $42 \mathrm{~m} 2$. Aquellos conjuntos de mayor escala (más de 250 viviendas), 11 en total, cuentan con equipamiento social (escuelas primarias y secundarias, centro de salud y posta policial) y un "arco de entrada" al barrio. Fueron denominados como "barrios-ciudades" (ejemplo, Ciudad Sol Naciente, Ciudad Evita, etc.).

En trabajos anteriores se han analizado las condiciones habitacionales de las familias que habitan los barrios-ciudades, caracterizadas en un alto porcentaje por situaciones de hacinamiento, que se relaciona directamente con las dimensiones de la unidad (2 dormitorios), planificada según los parámetros de familia nuclear tradicional, y la falta de previsión de un crecimiento flexible, acompañada por una política de financiamiento asequible para estos hogares (MARENGO Y ELORZA, 2018; MONAYAR, 2019; MARENGO, et.al., 2019).

Si bien este programa representó una de las acciones de mayor escala para el abordaje de los territorios informales en la ciudad de Córdoba, ello no significó una disminución en la reproducción de ocupaciones de tierra. A poco tiempo de entregadas las viviendas, fue posible advertir la aparición de situaciones de informalidad urbana en seis de estos conjuntos habitacionales (Figura 1), en un escenario marcado por el sostenido aumento del valor de los terrenos, la escasez de tierra urbanizada al alcance de las familias de bajos ingresos y la disponibilidad de suelo próximo a la localización de los conjuntos habitacionales públicos (MARENGO Y ELORZA, 2018; ELORZA, 2019; MONAYAR, 2019). 


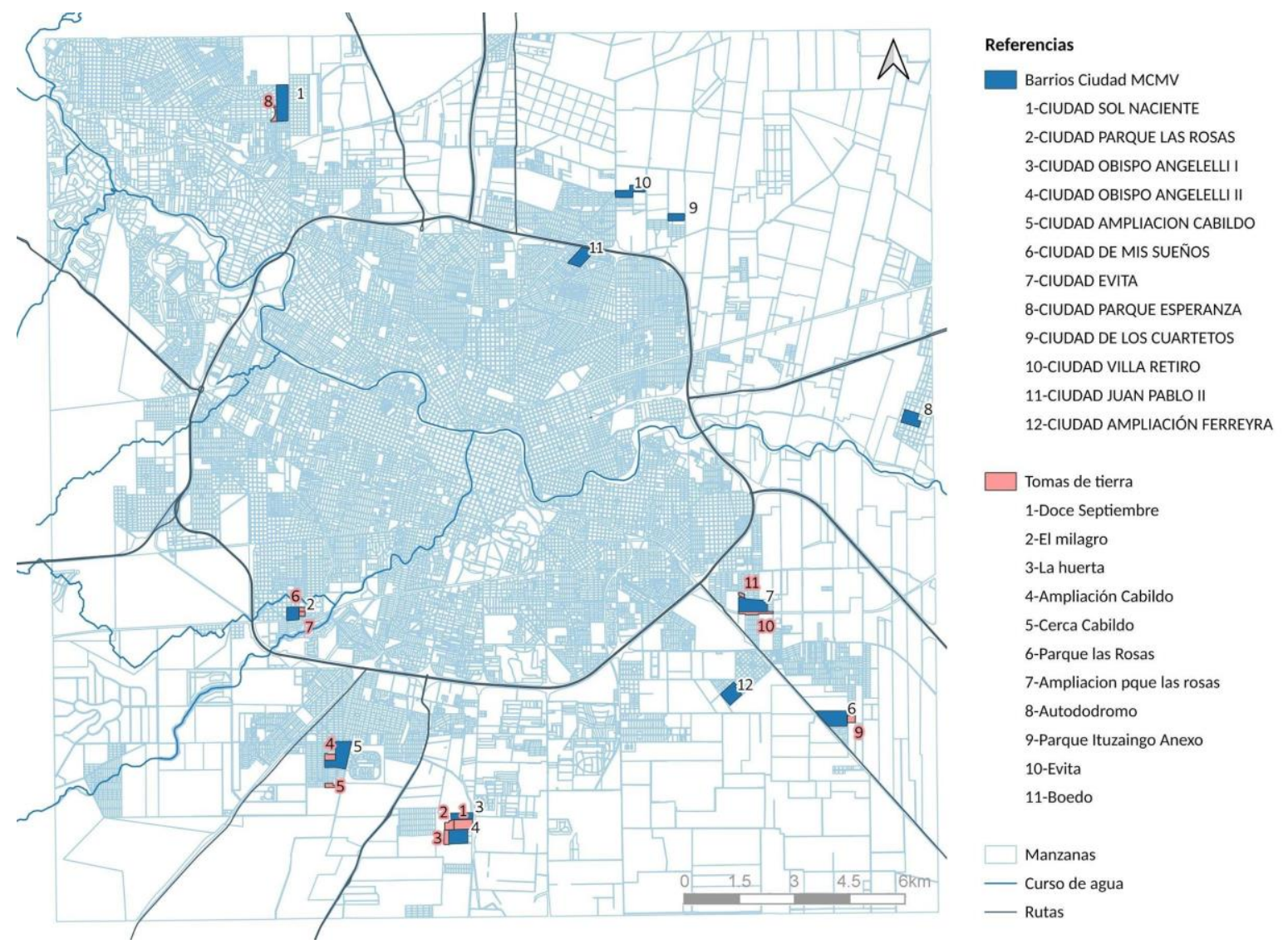

Figura 1: Localización de barrios-ciudades del Programa Mi Casa Mi Vida en Córdoba y tomas de tierra en colindancias. Elaboración propia. Fuente: Gobierno de la Provincia de Córdoba.

El presente trabajo se centra en el estudio de una de estas ocupaciones de tierra (Parque Ituzaingó Anexo II) colindante con el barrio Ciudad de Mis Sueños, al sureste de la ciudad de Córdoba.

\subsection{Ciudad de Mis Sueños y la configuración de nuevas demandas habitacionales}

Uno de los barrios ejecutados mediante el mencionado programa es "Ciudad de Mis Sueños". Se encuentra localizado en la zona sureste de la ciudad, en un sector en el que las urbanizaciones residenciales están desarticuladas dentro de un contexto rural- industrial. Fue inaugurado en junio del año 2004, está conformado por 565 viviendas unifamiliares y presenta una entrada demarcada por un arco en el que se expone su nombre y desde el que se accede a la calle pavimentada por la que circula el transporte público (Figura 2). 


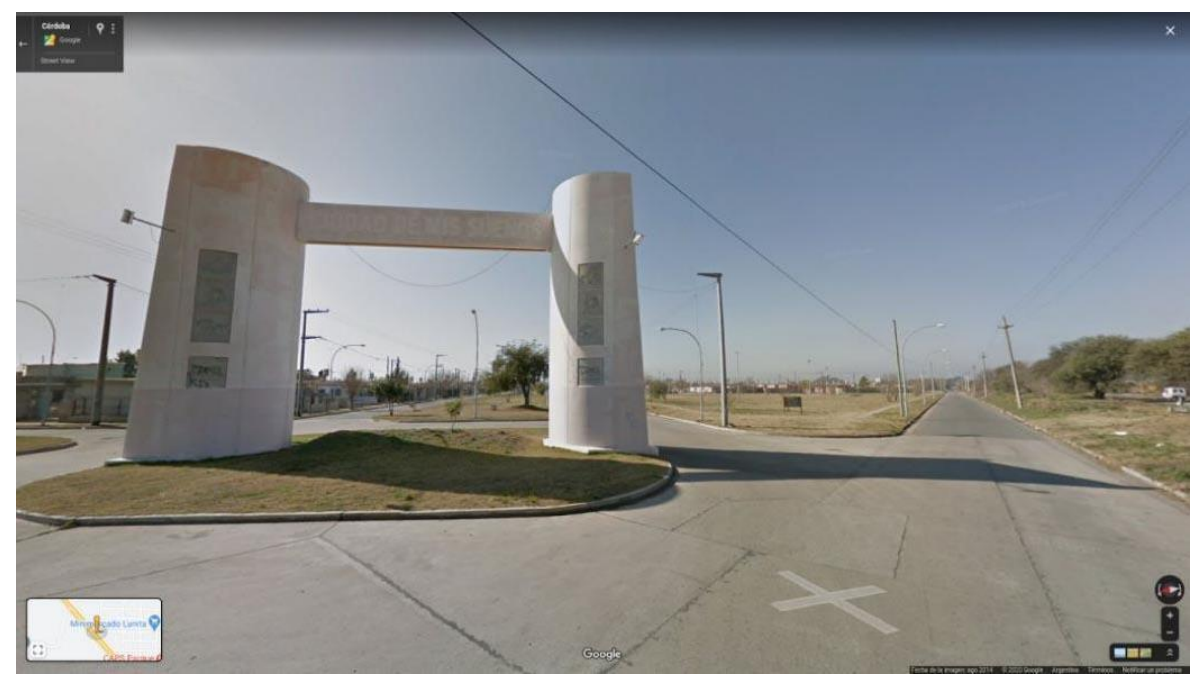

Figura 2: Arco de ingreso barrio Ciudad de Mis Sueños. Fuente: Google Maps

Las familias adjudicatarias provenían de villas localizadas en áreas centrales o consolidadas de la ciudad, por lo que la nueva localización periférica implicó importantes cambios y transformaciones en las estrategias de reproducción cotidiana, en especial, el mayor tiempo y costos para el traslado a los lugares de trabajo. Datos del Censo de 2010 indican que la población en Ciudad de Mis Sueños era de 2.147 habitantes, organizados en 511 hogares, de los cuales el 46,6\% tenían jefatura femenina. En relación a las condiciones de vida, se identifica que el 16,6\% de los hogares tenían Necesidades Básicas Insatisfechas (NBI). Los indicadores con mayor incidencia en esta medición son: el hacinamiento (60 hogares) y las condiciones sanitarias precarias (16 casos). Las situaciones de hacinamiento se relacionan directamente con las dimensiones de la tipología de vivienda planificada según los parámetros de familia nuclear tradicional.

En las observaciones realizadas en el barrio se pudo identificar como estrategia habitacional la densificación de los lotes, a través de la construcción de una unidad habitacional en el patio de las viviendas originales, para resolver cuestiones de hacinamiento y acceso a una segunda vivienda. Otro indicador de NBI presente en estos conjuntos habitacionales se relaciona con las condiciones sanitarias, dado que la ampliación si bien puede resolver el hacinamiento, no incluye sanitarios. Se presenta en el $3 \%$ sobre el total de hogares. Los indicadores de hacinamiento y condiciones sanitarias deficitarias dan cuenta de la condición de pobreza y escasos recursos económicos de los hogares, que imposibilita la construcción de nuevos espacios aun cuando se dispone de un lote amplio, y demuestra las dificultades que persisten para poder ampliar el espacio habitable mínimo que necesitan para el desarrollo de sus actividades cotidianas (MARENGO y ELORZA, 2018). 


\subsection{Informalidad derivada. El caso de Parque Ituzaingó anexo II}

Como mencionamos anteriormente, en las tierras colindantes a los barrios ciudades, se han desarrollado estrategias de toma de tierra en terrenos ociosos, que representaban problemas ambientales y de inseguridad. La proximidad con el barrio urbanizado otorga la posibilidad de extensión de redes precarias de infraestructura, acceso a servicios, equipamientos y continuidad de las redes sociales, familiares y otras de subsistencia.

La producción del asentamiento Parque Ituzaingó Anexo se originó en el año 2014, a través de una toma de tierras por parte de un grupo de familias, en su mayoría residentes del barrio Ciudad de Mis Sueños. Esta estrategia de acceso al hábitat se produce a diez años de la inauguración del barrio-ciudad, como modalidad de respuesta a las necesidades de un lugar de residencia para las familias jóvenes, que se encontraban en condiciones de hacinamiento y precariedad habitacional y que compartían la vivienda con otros hogares. En este caso, la ocupación se realiza en un predio que colinda al este con la urbanización estatal y al norte con barrio Los Fresnos, otro asentamiento de origen informal, cuya ocupación ya fue regularizada (Figuras 3 y 4 ).

El inicio de este proceso estuvo marcado por conflictos derivados de la disputa en torno a la apropiación de la tierra, al poco tiempo un grupo de vecinos logró negociar con el titular del predio. El resultado fue la donación de una fracción de terreno con el compromiso de respetar sus límites y no expandir el asentamiento hacia todo el predio. Si bien, hasta el momento, el acuerdo no tiene formalización notarial, ha permitido cierta seguridad en la ocupación de la tierra. Por otra parte, en el año 2016 el Registro Nacional de Barrios Populares incorpora este asentamiento como parte de los 118 registrados en la ciudad de Córdoba, situación que también refuerza las condiciones de consolidación de la urbanización.

El asentamiento se organiza en cinco manzanas rectangulares, las calles internas mantienen una traza regular, son de tierra y, por momentos, son prolongaciones de la traza del barrio estatal. A partir del trabajo de relevamiento se identificaron 179 lotes, con superficies que varían entre 125 a 185 m2 en distintas situaciones de uso: 91 lotes están ocupados con edificaciones destinadas a viviendas, 3 con usos comerciales y comunitarios, 17 en proceso de construcción y 68 lotes están vacíos, sin edificar (Figura 5). El proceso de ocupación y configuración del asentamiento respeta los acuerdos establecidos con el titular del dominio, los límites impuestos en su extensión y la organización de la subdivisión de los lotes, aunque muchos se encuentren sin ocupación.

Por otra parte, a través de la organización colectiva de un grupo de hogares se ha iniciado la prolongación de las redes de electricidad y agua desde el barrio Ciudad de Mis Sueños disponiendo de 
ambos servicios de manera precaria. La proximidad con el "barrio ciudad" y otros del entorno, le confieren acceso al transporte público y otros servicios sociales de bienestar como: escuelas, salas cuna, centro de salud y comedores, entre otros.
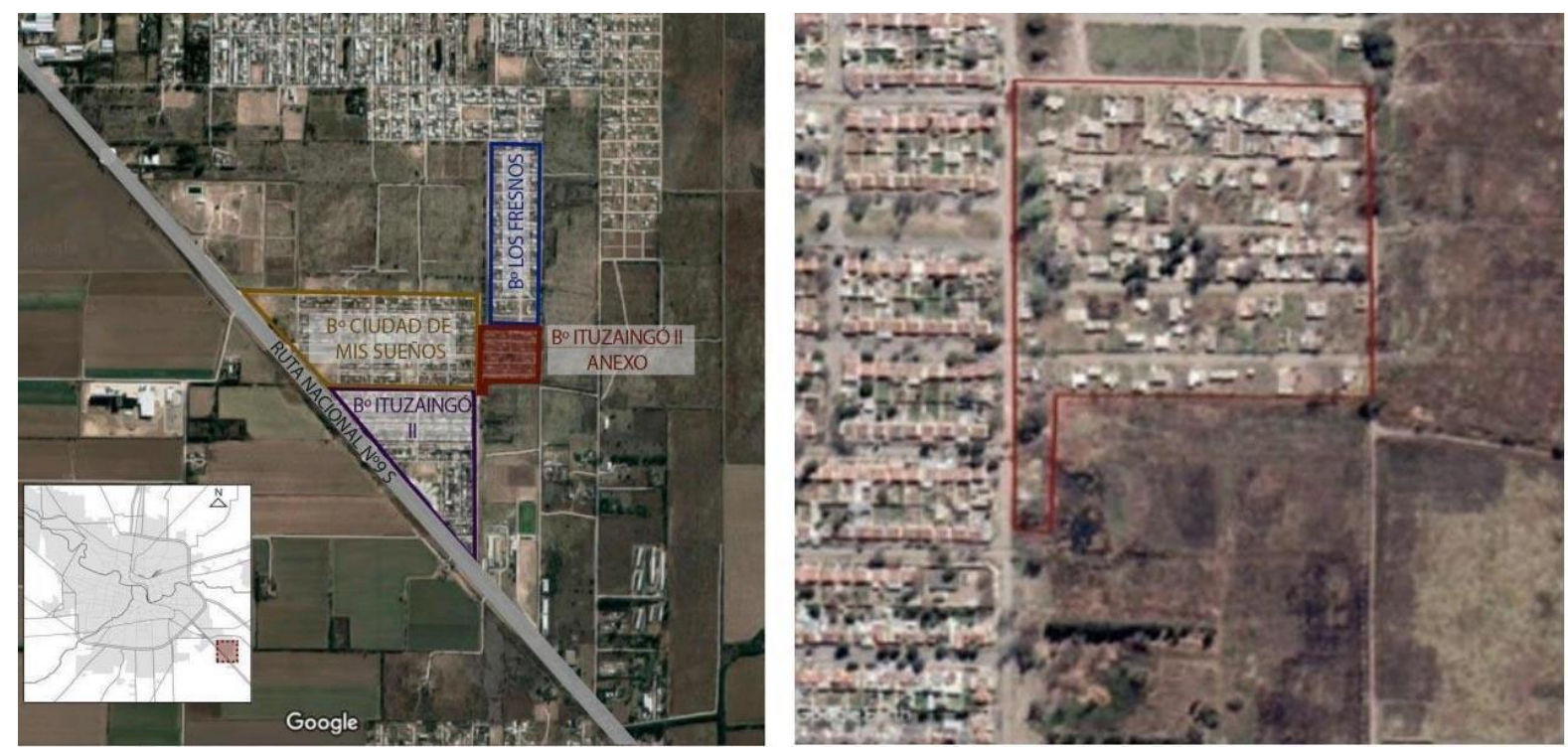

Figuras 3 y 4: Localización de la toma de tierra Parque Ituzaingó Anexo II, al oeste del barrio Ciudad de Mis Sueños.

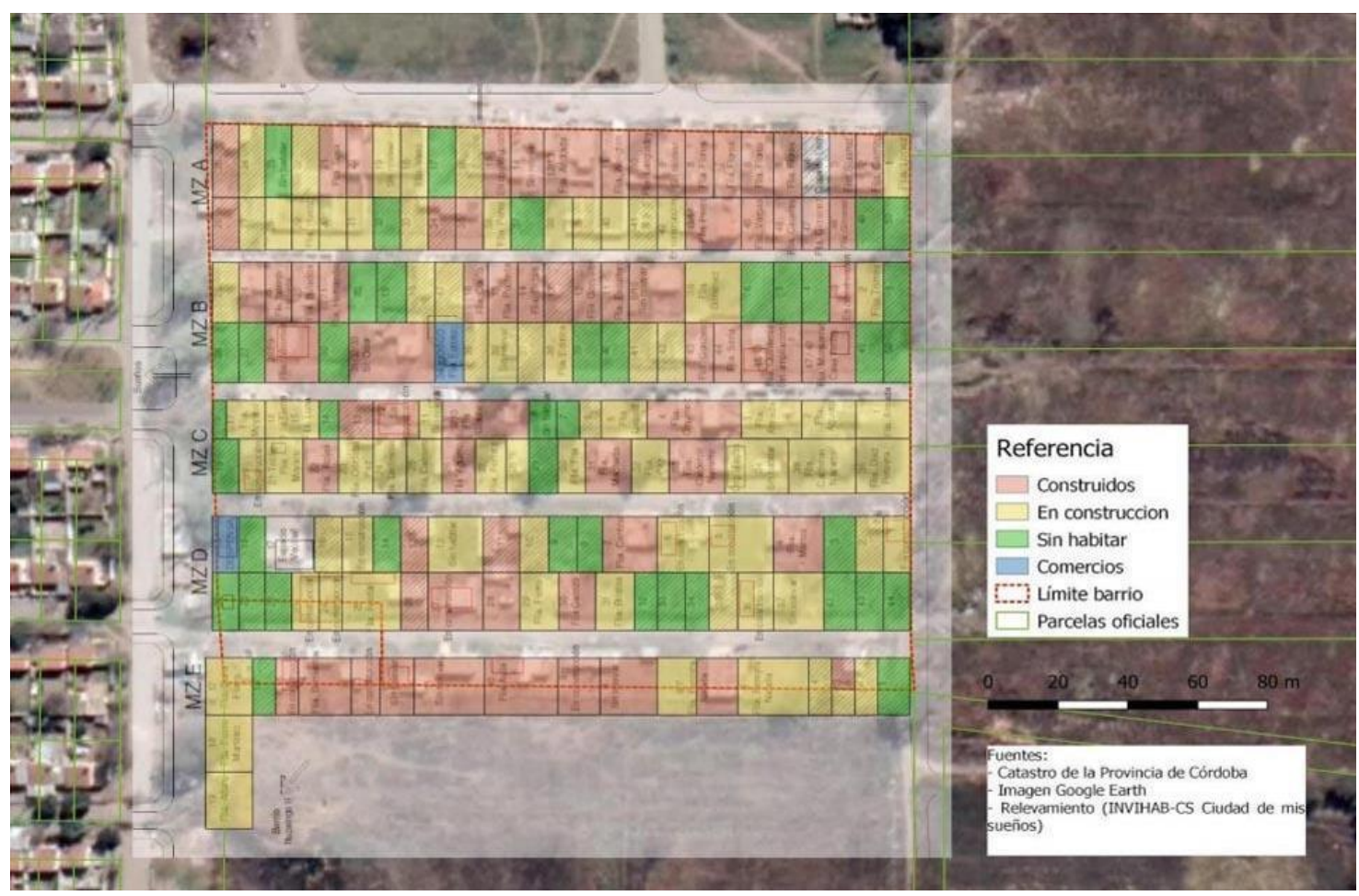

Figura 5: Conformación del asentamiento, manzanas y parcelamiento. Fuente: elaboración propia sobre la base del relevamiento realizado en campo. 

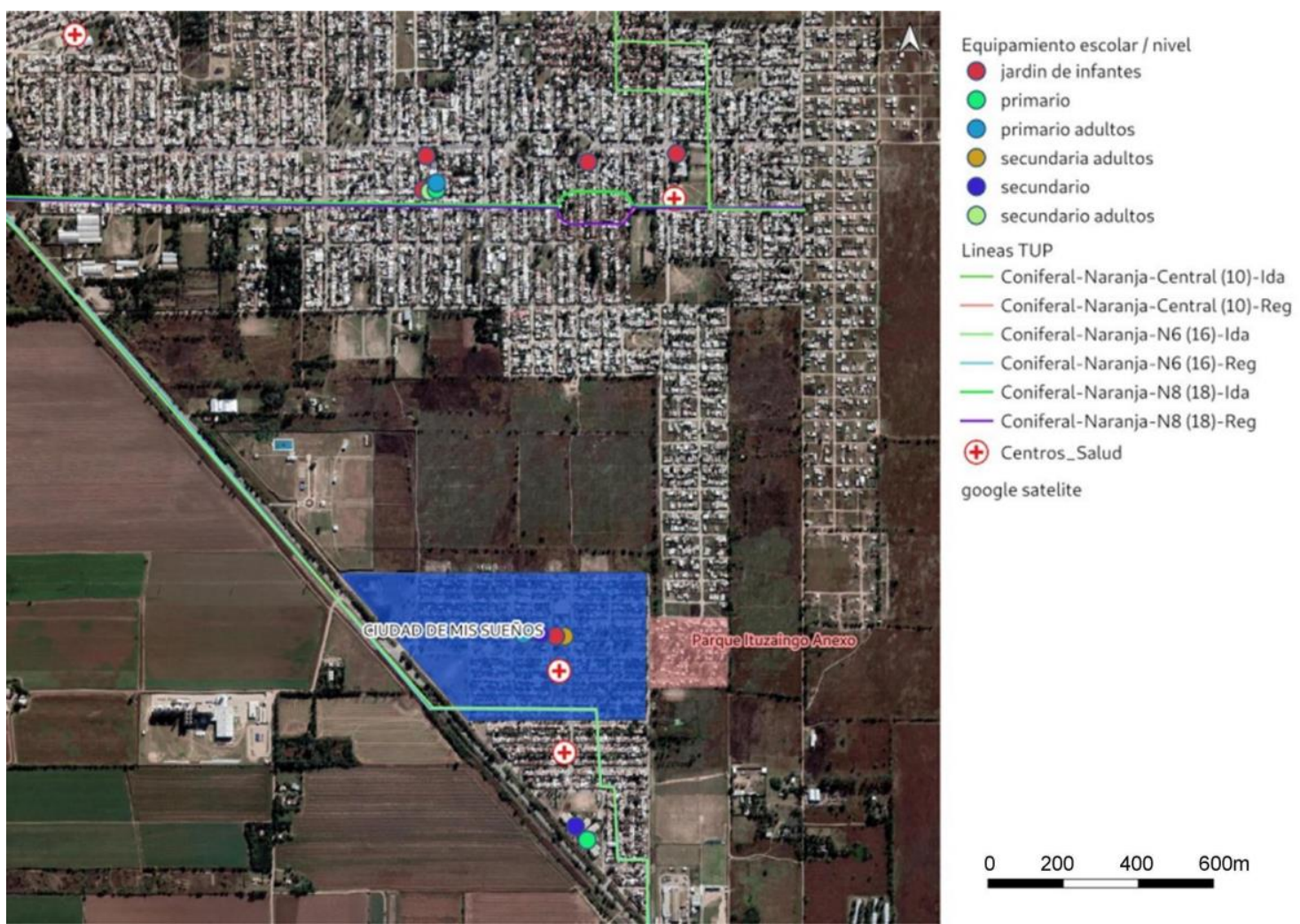

Figura 6: Relación con servicios en barrios próximos. Fuente: Elaboración propia en base a datos Municipalidad de Córdoba e IDECOR. Software QGis 3.10

\subsubsection{Características sociodemográficas}

Se identifica que la población total de residentes es de 262 personas. Demográficamente, se trata de una población relativamente joven, ya que el 92\% no supera los 45 años y el 51\% corresponde a niños, niñas y adolescentes. Otro dato a destacar, es la prevalencia de las mujeres en el rango etario de 19 a 30 años, que representa un $65,5 \%$ de las personas en ese grupo.

Se identificaron 66 hogares, de los cuales el $56 \%$ tienen jefatura masculina (37 casos) y el $44 \%$ restante, femenina (29 casos). La cantidad de personas por hogar es heterogénea, y se presentan diferentes tipos de organización familiar. Según la clasificación de tipos de hogar de INDEC, podemos identificar 3 hogares unipersonales y 63 multipersonales, donde se diferencian: 62 hogares multipersonales familiares (las personas que los componen están vinculadas por relaciones de parentesco) y 1 caso de multipersonales no familiares. En los hogares multipersonales familiares, se identifica una preponderancia de hogares nucleares con hijos $(60,6 \%)$ y hogares nucleares incompletos $20 \%$ (cuando no hay cónyuge, pero hay por lo menos un hijo del jefe/a). De este grupo, el 92\% corresponde a hogares con jefatura femenina, en su mayoría con hijos. 
Los resultados señalan que el $60 \%$ de los hogares están conformados por más de 4 miembros. Considerando que la media de la cantidad de personas por hogar para la ciudad de Córdoba es de 3,2, los datos del asentamiento dan cuenta de familias numerosas.

En relación al trabajo, el 38,4 \% de la población total del asentamiento está en edad económicamente activa (PEA de 14 a 60 años). De ese grupo, el 73,2 \% trabaja, el 11\% se encuentra desempleado/a, el $8,9 \%$ se encuentra inactivo (es decir, no busca trabajo) y sobre el $6.9 \%$ restante, no hay datos. Al analizar el total que trabaja, observamos que el $40 \%$ corresponde a trabajo no remunerado, en su mayoría refiere al trabajo intrafamiliar desarrollado por las mujeres. El $45 \%$ de las personas con inserción en el mercado ocupacional se desempeñan en trabajos informales, inestables y precarios, (por ejemplo, tareas en la construcción) y sólo el $15 \%$ está inserto en el mercado formal de trabajo con cobertura de seguridad social. Relacionando las categorías de inserción en el mercado de trabajo con el género, se observa que la mayor inserción en el trabajo remunerado es de los varones, pudiéndose inferir que sigue predominando una división sexual y social del trabajo dentro y fuera del hogar, quedando las mujeres a cargo del trabajo doméstico (cuidado y crianza de hijos/as).

Estos datos dan cuenta de las condiciones de vulnerabilidad socioeconómica de las familias del asentamiento, con frágiles soportes para la reproducción de la vida en un escenario marcado por la mercantilización de los bienes y servicios esenciales para la cotidianidad. Los datos demográficos reafirman esta condición, se trata de familias numerosas, jóvenes y con alta presencia de niños o menores, que ante la imposibilidad de acceder a una vivienda, participan en estrategias de toma de tierra y autoproducción del hábitat.

\subsubsection{Acceso a equipamientos y servicios sociales}

El acceso a los bienes y servicios colectivos por parte de los hogares para satisfacer distintas necesidades, está relacionado a la capacidad de cada uno de acceder, acumular y transferir recursos. En este sentido, para los hogares en condiciones de vulnerabilidad socioeconómica, el territorio barrial se constituye en un factor importante en el momento de la reproducción cotidiana, ya que en el mismo se establecen mecanismos de reconocimiento con otros sujetos e instituciones para el desarrollo de las prácticas de supervivencia (JELIN, 1998). En especial, hacemos referencia al acceso a los servicios dirigidos a establecer el nivel social mínimo de bienestar de la población en términos de salud y educación. Los datos del relevamiento indican que la población de niños/as y adolescentes del asentamiento tiene una importante inserción y permanencia en el sistema educativo: del grupo de 6 a 11 años, el 100\% se encuentra escolarizado/a; y del grupo de 12 a 17 años, el 78\% se asiste a la escuela, sólo el 22\% restante 
ha abandonado los estudios. Respecto a los establecimientos educativos a los que asisten, el $57 \%$ concurre a las instituciones educativas de Barrio Ciudad de Mis Sueños, el 36\% a las localizadas en barrio Ituzaingó (cercano al asentamiento) y el $7 \%$ restante acude a instituciones en otros sectores de la ciudad, desplazándose en transporte público.

En relación al acceso y cobertura sanitaria de la población, se resuelve en un 93\% en el sistema público de salud de jurisdicción provincial y/o municipal; el 7\% restante cuenta con cobertura por Obra Social (por su inserción en el mercado de trabajo como empleados formales). Las personas que residen en el asentamiento, en su mayoría acuden al primer nivel de atención próximo al espacio territorial de residencia, el 62,2\% de la población utiliza el Centro de Salud del barrio Ciudad de Mis Sueños, el 17,9\% acude a otros centros de salud de atención primaria del sector (de Bo Ituzaingó y al localizado en el Camino 60 cuadras) y otro $9,2 \%$ se traslada a hospitales de mayor complejidad. Del restante $10,7 \%$ de casos, no se cuenta con datos en relación a esta variable.

Si bien la localización del asentamiento es periférica, con déficits en relación a las vinculaciones con los sectores de centralidad y recursos urbanos, los equipamientos sociales (escuelas y centros de salud) que fueron materializados desde la política habitacional en el barrio Ciudad de Mis Sueños, constituyen los espacios de acceso a bienes y servicios esenciales para la reproducción de la vida cotidiana para estas familias.

\subsubsection{Producción del hábitat y condiciones habitacionales}

Los pobladores presentan heterogeneidad en las trayectorias habitacionales previas y los lugares de procedencia, como así también en los motivos de la participación en la ocupación informal y las formas de acceso a los lotes. Entre los motivos más recurrentes mencionados por la población, se identifican: la imposibilidad de afrontar los gastos del alquiler (38\%); el hacinamiento por hogar (31\% afirma compartir previamente la vivienda con otro hogar), y la única alternativa de acceso a un terreno propio (28\%).

Respecto a la procedencia, al momento de la toma de tierra la mayoría de las personas eran del barrio Ciudad de Mis Sueños y barrios aledaños, hogares jóvenes con perspectivas de acceder a un lote propio. Posteriormente, comenzó un proceso dinámico de llegada de nuevos hogares desarrollándose un mercado informal de acceso a los terrenos vacantes, con población proveniente de barrios próximos. Al momento del relevamiento, se identifica que el $51 \%$ de la población residente proviene de barrios cercanos al asentamiento (Ituzaingó, Los Fresnos y Los Sauces), el 34\% habitaban originalmente en barrio Ciudad de Mis Sueños, y el resto (26\%) consignan procedencia de diferentes barrios con una localización muy distante respecto al conjunto de vivienda social. Las modalidades de acceso al lote son variadas, es 
significativo el porcentaje de hogares (26\%) que formaron parte de la ocupación colectiva de la tierra en el momento de la "toma". Asimismo, se reconoce que, con el tiempo, la mayoría de las familias (62\%) realizaron la compra de los lotes a través del mercado informal en el asentamiento; y un $12 \%$ de familias se declaran ocupantes por préstamos o donación.

Sobre la producción de la vivienda, la mayoría de los hogares (82\%) refieren a la autoconstrucción, mientras que el $7 \%$ accedieron a la vivienda mediante la compra y el $11 \%$ restante lo hizo a través del alquiler, la donación o el préstamo.

En el caso de las edificaciones, se observan procesos de construcción progresiva, propio de los procesos de ocupación informal. Si bien originalmente las viviendas son precarias, rápidamente se consolidan con materiales sólidos y en superficie, atendiendo a la dinámica demográfica de las familias.

A partir de los datos relevados, se puede apreciar una relación entre el tamaño de la vivienda y el tiempo de residencia en el asentamiento; la mayoría de las viviendas con más de un dormitorio corresponden a hogares con más de dos años de residencia en el barrio, mientras que los hogares con menor tiempo de residencia habitan en monoambientes o unidades habitacionales con un solo dormitorio.

Cuando se analizan las características de las viviendas en relación al tamaño de los hogares (4 miembros), resulta que un $71 \%$ de casos presentan situaciones de hacinamiento crítico. Dado lo exiguo del núcleo habitacional y atendiendo a lo mencionado en el relevamiento, el espacio destinado a dormir, se reconoce en sólo 47 viviendas. A ello se agregan dos unidades de vivienda que presentan condiciones de hacinamiento por hogar.

La dotación de servicio de agua potable es precaria a través de la prolongación de mangueras (a veces bajo tierra) desde la urbanización estatal colindante. Esa situación se suma a las características de precariedad sanitaria en las viviendas. Si bien el $41 \%$ de casos, refieren poseer baño instalado, se identifica esta característica con la disponibilidad de un inodoro aún cuando la descarga de agua se realiza de forma manual, dado las condiciones de provisión de agua, y la imposibilidad de contar con tanque de reserva domiciliario para la descarga de los artefactos. Por otra parte, el $20 \%$ de los hogares refieren no disponer de baño en las viviendas y el 39\% cuenta con excusado o letrina. Se considera que la instalación completa y adecuada del baño es uno de los ítems con mayores costos asociados en la edificación y cualificación de la mano de obra, mientras que es uno de los indicadores contemplados en la medición de la pobreza estructural a través de las Necesidades Básicas Insatisfechas.

En relación a las consultas realizadas a los pobladores del asentamiento sobre las problemáticas prioritarias que advertían en su territorio, en el 60\% de casos manifiestan preocupación por la dotación 
de los servicios de agua y luz (60\%); tanto por la calidad del acceso a estos servicios básicos para la reproducción de la vida, como por los riesgos derivados de las conexiones precarias (en especial, referido al tendido eléctrico). También se advierten problemas ambientales, en la gestión de residuos y el uso como basural del terreno colindante al asentamiento.

\section{CONCLUSIONES}

A más de diez años de la implementación de un programa de vivienda social de fuerte impacto en la configuración urbana de la periferia de la ciudad por la cantidad de unidades habitacionales construidas y la escala de los conjuntos, (como lo fue el Mi Casa Mi Vida, en Córdoba) observamos que la forma de producción de los territorios periféricos no se ha modificado, continúa reproduciendo un espacio altamente fragmentado, desarticulado, con situaciones de informalidad y precariedad habitacional en las formas de acceso al suelo y la vivienda.

El caso del asentamiento Parque Ituzaingó, aporta a la comprensión de las lógicas que subyacen en los entornos construidos a partir de la intervención estatal, mostrando que la localización de borde de inicio, se ha constituido en oportunidad, aunque precaria, de acceso al suelo urbano por parte de las familias jóvenes, como estrategia de acceso al hábitat, en un escenario marcado por la ausencia de políticas habitacionales desde la materialización del programa a la fecha. En este caso, el barrio Ciudad de Mis Sueños es el soporte que facilita el acceso a los servicios (transporte, salud, educación), a los equipamientos sociales y la extensión (informal) de las redes de agua y electricidad. Demuestra, además, cómo los entornos de la política habitacional se convierten en los nuevos espacios de la informalidad, reproduciendo situaciones de carencia que la propia acción de la política se proponía resolver, dada la ausencia de políticas para acompañar las nuevas demandas habitacionales en el transcurrir temporal de los diez años.

En este sentido, el análisis realizado permite comprender los aspectos que desencadenan la toma de tierra y el posterior proceso de autoproducción del hábitat en los bordes del conjunto producido por la política pública habitacional; constituyéndose una estrategia resultante de múltiples factores como: las condiciones socio-demográficas de la población adjudicataria de las viviendas sociales, la persistencia de condiciones de pobreza y situaciones de vulnerabilidad de los habitantes de estos barrios, las redes sociales establecidas en el territorio, la creciente demanda de un lugar de residencia por parte de esta población, sumado a las características de la localización y la colindancia con áreas de suelo vacante. 
Cabe destacar que el rol del Estado en los procesos de autoproducción del hábitat desde lógicas informales no es uniforme, sino que varía en relación a la localización, los actores e intereses involucrados y en las relaciones que se establecen. Las tomas de tierra son toleradas (incluso a veces promovidas y aceptadas por los mismos propietarios de la tierra), omitidas, desalojadas y, en ciertos casos también toleradas por el mismo Estado, procurando mantener el control y negociando con sus habitantes la resolución de las necesidades con un amplio rango de discrecionalidad. El caso del asentamiento analizado, que inicialmente estuvo marcado por conflictos derivados de su judicialización con el titular del dominio y posteriormente derivó en una donación de parte del predio ocupado, revela los procesos de negociación entre los actores en relación al acceso al suelo y la posterior consolidación de la ocupación informal. También, muestra las limitaciones de los mismos, dado que se trata de arreglos de palabra, que no han sido formalizados ante los organismos técnicos del Estado. Esta situación impide avanzar en la regularización para el acceso a los servicios (como es el caso de las redes de agua potable y electricidad), lo cual contribuye a sostener procesos deficitarios en las condiciones habitacionales, en la calidad de vida de las familias y en sus derechos como ciudadanos.

El proceso de conformación de los paisajes para la vida, da cuenta de la dinámica de estos territorios y la persistencia de condiciones de vulnerabilidad y precariedad habitacional, más allá de la potencialidad y capacidad de autoproducción del hábitat por parte de las familias. Los datos resultantes del relevamiento demuestran las necesidades habitacionales que no se agotan en la vivienda como hecho físico, aunque ésta sea el disparador de las acciones de ocupación del suelo, y se inscriben en el contexto más amplio de la producción urbana y las posibilidades de acceso a servicios, equipamiento e infraestructura en contexto de alta inequidad.

Repensar un tipo de política habitacional que atienda las situaciones de urbanización informal, implica considerar una escala de intervención territorial que no quede limitada a la materialización de un conjunto habitacional, sino que pueda intervenir planificando la evolución de un área de mayor escala, previendo la necesidad de futuras urbanizaciones para contemplar condiciones de acceso al suelo y la vivienda. El reconocimiento de la capacidad de los pobladores para desarrollar procesos de autogestión del hábitat es un indicador que podría ser incorporado en el diseño de futuras políticas habitacionales, orientadas a mejorar las condiciones de la vivienda y de los recursos urbanos (servicios, equipamientos y movilidades) necesarios para la efectivización del derecho a la ciudad. 


\section{BIBLIOGRAFÍA}

ACIOLY, Claudio (2018) Vivienda y urbanización sustentable e inclusiva en la nueva agenda urbana. Vivienda \& Ciudad n. 5, Córdoba, pp. 28-35. Recuperado de: https://revistas.psi.unc.edu.ar/index.php/ReViyCi/article/view/22797

ABRAMO, Pablo (2012). La ciudad com-fusa: Mercado y producción de la estructura urbana en las grandes metrópolis latinoamericanas. EURE, n. 38(114), Santiago de Chile, pp. 35-69. https://doi.org/10.4067/S0250- 71612012000200002

CARRION, Fernando y Hanley, Lisa (2005) Regeneración y revitalización urbana en las Américas: hacia un Estado estable. Flacso Ed, Quito.

CATTENAZZI, Andrea y REESE, Eduardo (2016) Argentina. A 20 años de la Hábitat II, las asignaturas pendientes. En: Cohen, M., Carrizosa, M. y Gutman, M. (Eds.) Hábitat en deuda. 20 años de políticas urbanas en América Latina (pp. 295-372). Ed. Café de las Ciudades Colección Hábitat, Buenos Aires.

CRAVINO, María Cristina (2006) Las villas de la ciudad: mercado e informalidad urbana. Ed. Universidad Nacional General Sarmiento, Buenos Aires.

DE MATTOS, Carlos y LINK, Felipe (2015) Lefebvre revisitado: capitalismo, vida cotidiana y el derecho a la ciudad. Ril Editores Colección Estudios urbanos Universidad Católica, Santiago de Chile.

DUHAU, Emilio y GIGLIA, Angela (2008) Las reglas del desorden: habitar la metrópoli. Siglo XXI Editores, Ciudad de México.

ELORZA, Ana Laura (2019) Segregación residencial y estigmatización territorial. Representaciones y prácticas de los habitantes de territorios segregados. EURE, 45 (135), Santiago de Chile, pp. 91-109. http://dx.doi.org/10.4067/S0250-71612019000200091

ELORZA, Ana Laura y MORILLO, Ernesto (2017) El territorio urbano en disputa: representaciones y prácticas en procesos de autoproducción del hábitat. Conciencia social, n. 1, Córdoba, pp. 28-46. https://revistas.unc.edu.ar/index.php/ConCienciaSocial/issue/view/1521

HARVEY, David (2013) Ciudades Rebeldes. Del derecho a la ciudad a la revolución urbana. Akal, Buenos Aires.

HIDALGO DATTWYLER, Rodrigo (2007) ¿Se acabó el suelo en la gran ciudad? Las nuevas periferias metropolitanas de la vivienda social en Santiago de Chile. EURE, 33 (98), Santiago de Chile, pp. 57-75. Recuperado de http://www.eure.cl/index.php/eure/article/view/1351

---(2019) Políticas de vivienda social y la Precariopolis estatal. Conferencia Flacso Ecuador, 04 de febrero, archivo en MP3, 00:47:34. Recuperado de:

https://repositorio.flacsoandes.edu.ec/handle/10469/15458

HIDALGO DATTWYLER, Rodrigo y JANOSCHKA, Michael (2014) La ciudad neoliberal: estímulos de reflexión crítica. En Hidalgo, R. y Janoschka, M. (Eds.) La ciudad neoliberal. Gentrificación y exclusión en Santiago de Chile, Buenos Aires, Ciudad de México y Madrid (pp. 7-32). Pontificia Universidad Católica de Chile, Santiago de Chile. 
JELIN, Elizabeth (1998) Pan y Afectos. La transformación de las familias. Fondo de Cultura Económica, Buenos Aires.

MARENGO, Cecilia, ELORZA, Ana Laura y SOSA, Florencia (2019) Transformaciones territoriales, políticas públicas y espacios periféricos. Conjuntos de vivienda social en Córdoba (Argentina) a una década de su materialización. SIIU XI Barcelona- Santiago de Chile, n. 11 (pp. s/n) http://dx.doi.org/10.5821/siiu.6784

MARENGO, Cecilia y ELORZA, Ana Laura (2018) Segregación residencial socioeconómica y programas habitacionales públicos: el caso del programa Mi casa, Mi vida en la ciudad de Córdoba, Argentina. Direito da Cidade n.10 (3), Brasil, pp.1542-1568 http://dx.doi.org/10.12957/rdc.2018.32765

MONAYAR, Virginia (2018) Ocupación Informal Del Espacio Urbano. Situaciones, Características y factores determinantes, en el Municipio de Córdoba, Argentina 1990-2010. ACE: Architecture, City and Environment $=$ Arquitectura, Ciudad y Entorno, n.12 (36), Barcelona, pp. 111-130.

http://dx.doi.org/10.5821/ace.12.36.4800

MONAYAR, Virginia, ALVARADO RODRIGUEZ, Mónica y ROJAS, Mara (2019) Entre lo formal y lo informal. Las tomas de tierra al "margen" de la política habitacional en Córdoba, Argentina. Revista F@ro, n. 2 (30), Valparaíso, pp. 33-53. Recuperado de https://www.revistafaro.cl/index.php/Faro/article/view/602/555

ORTIZ, Eduardo (2007) Integración de un sistema de instrumentos de apoyo a la producción social de la vivienda. Coalición Internacional por el Hábitat, México.

RODRÍGUEZ, Carla, DI VIRGILIO, Mercedes, PROCUPEZ, Valeria, VIO, Marcela, OSTUNI, Fernando; MENDOZA, Mariana y MORALES, Betsy (2007) Producción social del hábitat y políticas en el Área Metropolitana de Buenos Aires: historia con desencuentros. Recuperado de: http://biblioteca.clacso.edu.ar/Argentina/iigg-uba/20100720101204/dt49.pdf

RODRÍGUEZ, Alfredo y RODRÍGUEZ, Paula (2016) Chile. Vivienda privada de ciudad. En Cohen M., Carrizosa M. y Gutman M. (Eds.) Hábitat en deuda. Veinte años de políticas urbanas en América Latina, pp 101-152. Café de las ciudades, Buenos Aires.

RODRÍGUEZ, Alfredo y SUGRANYES, Ana (2005) Los con techo. Un desafío para la política de vivienda social. Ediciones SUR, Santiago de Chile.

ROLNIK, Raquel (2018) Prólogo. En Hernández, M. y Diaz Garcia V. (Coord.) Visiones del hábitat en América Latina (pp. 7-11). Ed. Reverte, Madrid.

ROY, Ananya (2013) Las metrópolis del Siglo XXI nuevas geografías de la teoría. Andamios n. 10 (22), Buenos Aires, pp.149-182. Recuperado de: https://andamios.uacm.edu.mx/index.php/andamios/article/view/271

SECCHI, Bernardo (2015) La ciudad de los ricos y la ciudad de los pobres. Ed. Catarata, Madrid.

SOJA, Edward (2014) En busca de la justicia espacial. Tirant Humanidades, Valencia. 
SVAMPA, Maristella y VIALE, Eduardo (2014) Maldesarrollo. La Argentina del extractivismo y el despojo. Katz, Buenos Aires.

ZICCARDI, Alicia (2015) Cómo viven los mexicanos. Análisis regional de las condiciones de habitabilidad de la vivienda. Universidad Nacional Autónoma de México, Instituto de Investigaciones Jurídicas, México.

Trabalho enviado em 19 de fevereiro de 2021

Aceito em 22 de fevereiro de 2021 